\section{SCIENCE CHINA \\ Physics, Mechanics \& Astronomy}

- Editorial•

Special topic: New advances in water and water systems
October 2019 Vol. 62 No. 10: 107001

https://doi.org/10.1007/s11433-019-9432-3

\title{
Preface to the special topic: New advances in water and water systems
}

Water and related systems play a central role in science and technology. Although water has unusual and anomalous behaviors, if compared with normal liquids, it is of fundamental interest in many research fields going from chemical-physics to life sciences. The first and well known example of these anomalies is the density maximum, at $277 \mathrm{~K}$, intuited by Galilei [1] in 1612 and lately discovered in Florence [2].

These anomalies are present in both the solid and liquid forms. In fact, from one side the crystalline ice phase diagram, as discovered by Bridgman [3] in 1912, is characterized by the polymorphism. From the other side the liquid, depending on pressure and temperature, shows a complex behavior in its thermodynamic response functions (and the corresponding fluctuations) in particular inside the metastable supercooled state. A reason for which water is considered a prototype of complex materials and glass forming liquids; it in fact, can be cooled below its melting temperature $\left(T_{\mathrm{m}}\right)$ up to the homogeneous nucleation temperature.

The difference between a normal liquid and water is just in the fluctuations behaviors; in a normal fluid both the entropy and volume fluctuations become smaller and positively correlated as $T$ decreases, on the contrary in water they become more pronounced and below $T_{\mathrm{m}}$ are anti-correlated (a volume increase brings an entropy decrease). Hence the water cooling is accompanied by a pronounced and increasing local order, due to the Hydrogen bond (HB) interaction, inside the supercooled state also resulting in a diverging (critical like) behavior of the thermodynamic functions. As proposed by Speedy and Angell [4], at ambient pressure the specific heat and the compressibility appear to diverge at a singular temperature $T_{\mathrm{s}} \sim 228 \mathrm{~K}$.

At the beginning of the 1960s, just in order to account for water anomalous properties, experimental and theoretical studies suggest the idea of "two liquids of the same substance". Just in 1967 Rapoport [5] wrote "Several models postulating the existence of two species have been proposed in order to account for the anomalous properties of water." Only after the Mishima's discovery of the amorphous ice polymorphism in 1985 [6], namely the low and high density glasses, such an idea was enforced. Few years later these experimental findings, in 1992, in a computational study the Stanley's team provided an appropriate link between the glass and liquid polymorphism suggesting a proper explanation for the anomalous behavior of the supercooled water's response functions [7]. In particular, such a simulation was coherent with the occurrence of a first-order phase transition between two liquids, whose line extends into the solid amorphous region.

This volume, reporting some of the contributions given at the International School "Water and Water Systems-The Hydrophobic Effect" held in ERICE (Italy) from 4 to 11 July 2018 at the Ettore Majorana Foundation and Centre for Scientific Culture, gives an overview of the present status of the water physics encompassing experiments, theory and computation. Transport properties as well as phase behaviors; hydrophilic interactions as well as hydrophobic, of water and water systems are discussed [8-18].

Our sincere thanks go to many. First and foremost to those lecturers who put extra efforts to make their presentation a model of pedagogical approach to science, to those students whose frequent questions and perceptive observations opened new direction of pursuit, to the members of the Ettore Majorana Foundation Center for their 24-hour days of managerial helps and lightness of heart that created a relaxed atmosphere conductive to intellectual stimulation. Unfortunately, it was of course impossible to reproduce in this volume the lively and friendly atmosphere of the school, especially in the San Rocco garden after the dinner, enriched by the Erice almond cakes and Marsala wine.

We are particularly grateful for the generous support to the Messina, Naples Federico II and Palermo Universities, the 
Consiglio Nazionale delle Ricerche and the Consorzio Internazionale per lo Sviluppo di Sistemi a Grande Interfase. It is also a pleasure to record our immense debit to the MIFT Department of the Messina University and to the Department of Physics and Chemistry of the Palermo University for the many helps and encouragements. We also wish to thank the vital contribution of Profs. A. Cupane and E. Fazio, and of Drs. C. Corsaro, G. Cottone and D. Mallamace as members of the organizing committee. Lastly, but certainly not the last, we greatly appreciate the generous support of SCIENCE CHINA Physics, Mechanics \& Astronomy and the editor Dr. Wei Wang for the publication of this special topic.

\author{
LiMei $\mathrm{Xu}^{1}$, and Francesco Mallamace ${ }^{2}$ \\ ${ }^{1}$ School of Physics, Peking University, Beijing 100871, China; \\ ${ }^{2}$ Dipartimento di Fisica, Università di Messina, Messina I-98166, Italy
}

1 G. Galilei, Discorso al Serenissimo Don Cosimo II Gran Duca di Toscana: Intorno Alle Cose, Che Stanno in sù L'Acqua, ò Che in Quella si Muovono (Cosimo Giunti, Florence, 1612).

2 L. Magalotti, Saggi di naturali esperienze fatte nell'Accademia del Cimento sotto la protezione del Serenissimo principe Leopoldo di Toscana e descritte dal segretario di essa accademia (Giuseppe Cocchini all' Insegna della stella, Florence 1667, Esperienze intorno agli artificiali agghiacciamenti), pp. 127-176.

3 P. W. Bridgman, Proc. Am. Acad. Arts Sci. 47, 441 (1912).

4 R. J. Speedy, and C. A. Angell, J. Chem. Phys. 65, 851 (1976).

5 E. Rapoport, J. Chem. Phys. 46, 2891 (1967).

6 O. Mishima, L. D. Calvert, and E. Whalley, Nature 314, 76 (1985).

7 P. H. Poole, F. Sciortino, U. Essmann, and H. E. Stanley, Nature 360, 324 (1992).

8 C. Zhang, Y. Yang, and P. Zhang, Sci. China-Phys. Mech. Astron. 62 , 107002 (2019).

9 D. Mallamace, S.-H. Chen, C. Corsaro, E. Fazio, F. Mallamace, and H. E. Stanley, Sci. China-Phys. Mech. Astron. 62, 107003 (2019).
10 F. Mallamace, C. Corsaro, D. Mallamace, S.-H. Chen, E. Fratini, and P. Baglioni, Sci. China-Phys. Mech. Astron. 62, 107004 (2019).

11 F. Mallamace, C. Corsaro, E. Fazio, S. H. Chen, and D. Mallamace, Sci. China-Phys. Mech. Astron. 62, 107005 (2019).

12 R. Pastore, M. Caggioni, D. Larobina, L. S. Amato, and F. Greco, Sci. China-Phys. Mech. Astron. 62, 107006 (2019).

13 A. Fierro, A. de Candia, and A. Coniglio, Sci. China-Phys. Mech. Astron. 62, 107007 (2019).

14 C. Andreani, C. Corsaro, D. Mallamace, G. Romanelli, R. Senesi, F. Mallamace, Sci. China-Phys. Mech. Astron. 62, 107008 (2019).

15 T. O. Fonseca, M. M. Szortyka, P. Ternes, C. Gavazzoni, M. C. Barbosa, Sci. China-Phys. Mech. Astron. 62, 107009 (2019).

16 I. Zhovtobriukh, B. J. C. Cabral, C. Corsaro, D. Mallamace, and L. G. M. Pettersson, Sci. China-Phys. Mech. Astron. 62, 107010 (2019).

17 A. Iorio, G. Camisasca, and P. Gallo, Sci. China-Phys. Mech. Astron. 62, 107011 (2019).

18 V. D. Michele, G. Romanelli, and A. Cupane, Sci. China-Phys. Mech. Astron. 62, 107012 (2019). 